\title{
DIFFERENTIAL OPERATORS AND THETA SERIES
}

\author{
BY \\ SOLOMON FRIEDBERG ${ }^{1}$
}

\begin{abstract}
Let $f$ be a modular form on a congruence subgroup of $\operatorname{SL}(2, \mathbb{Z})$ - not necessarily holomorphic, but an eigenfunction of the weight $k$ Casimir operator. Maass introduced differential operators (coming from the complexified universal enveloping algebra) which raise and lower by 2 the weight of such a form and shift the eigenvalue. Here we introduce differential operators on hyperbolic 3 space analogous to the Maass operators. These change by 2 the weight of a modular form for an imaginary quadratic field.

THEOREM. The Maass operators and the hyperbolic space operators are intertwined by the imaginary quadratic Doi-Naganuma (base change) lifting. That is, the following diagram is commutative:
\end{abstract}

\begin{tabular}{|c|c|c|c|}
\hline & $F$ & $\begin{array}{c}\text { hyperbolic space } \\
\leftrightarrow \\
\text { operators }\end{array}$ & $\tilde{H}$ \\
\hline Lift & $\mathfrak{\imath}$ & & \\
\hline & $f$ & $\begin{array}{c}\text { Maass } \\
\stackrel{\leftrightarrow}{\text { operators }}\end{array}$ & \\
\hline
\end{tabular}

\begin{abstract}
Using similar techniques for the dual pair $(\operatorname{SL}(2, \mathbf{R}), \operatorname{SO}(2,1))$, we give a simple proof that the Shimura correspondences preserve holomorphicity (for weight $\geqslant 5 / 2$ ) and an explanation for this property directly in terms of the theta series (Weil representation) integral kernel. We also establish similar results for the real quadratic Doi-Naganuma lifting.
\end{abstract}

Introduction. Correspondences between automorphic forms on certain groups, obtained by integrating against theta series, have been much studied. For example, when one of the groups in question is $\operatorname{SL}(2, \mathbb{R})$, various cases have been examined by Niwa [9] and Shintani [13] (the Shimura correspondence), Kudla [7] (the real quadratic Doi-Naganuma map), and Asai [1] and myself $[3,4]$ (the imaginary quadratic Doi-Naganuma map). In this paper we shall study the interplay between these correspondences and certain differential operators on spaces of automorphic forms. This interplay provides a natural explanation for the holomorphicity properties of the Shimura and real quadratic Doi-Naganuma maps, and, in the imaginary quadratic Doi-Naganuma case, leads to the study of certain new differential operators on hyperbolic 3-space, which seem to us to be of independent interest.

Received by the editors September 19, 1983 and, in revised form, February 7, 1984. A preliminary version of this work was presented in the session on Automorphic Forms and Automorphic Representations at the AMS Summer Meeting in Albany, New York, on August 8, 1983.

1980 Mathematics Subject Classification. Primary 10D15; Secondary 10D20, 12 A67.

Key words and phrases. Theta function, modular form, Doi-Naganuma lifting, Shimura correspondence, Maass operator, hyperbolic 3-space.

${ }^{1}$ Research supported in part by a National Science Foundation Postdoctoral Research Fellowship. 
To explain our results and the paper's organization more fully, let us introduce the following notation. By a modular form of weight $k$ (an integer) and eigenvalue $\lambda$ on a subgroup $\Gamma$ of $\operatorname{SL}(2, \mathbb{Z})$, we shall mean a function $f$ from the upper half plane

$$
\mathfrak{S}=\{z \in \mathbb{C} \mid \operatorname{Im}(z)>0\}
$$

to $\mathbb{C}$ such that:

(1) $f(\gamma z)=(c z+d)^{k} f(z)$ for all $\gamma=\left(\begin{array}{ll}a & b \\ c & d\end{array}\right)$ in $\Gamma$;

(2) $f(z)$ has (at worst) polynomial growth at each cusp (in the usual sense); and

(3) $\Omega^{k, \lambda} f=0$,

where

$$
\Omega^{k, \lambda}=-y^{2}\left(\frac{\partial^{2}}{\partial x^{2}}+\frac{\partial^{2}}{\partial y^{2}}\right)+2 i k y \frac{\partial}{\partial \bar{z}}+\lambda \quad\left(\frac{\partial}{\partial \bar{z}}=\frac{1}{2}\left(\frac{\partial}{\partial x}+i \frac{\partial}{\partial y}\right)\right) .
$$

(cf. §1). We write the space of such $f$ as $\{\Gamma, k, \lambda\}$. There are differential operators $\mathbf{D}_{k}^{r}$ and $\mathbf{D}_{k}^{1}$, introduced by Maass [8], which act on $\{\Gamma, k, \lambda\}$, raising and lowering (respectively) the weight of a modular form by 2 , and shifting its eigenvalue. We shall study the action of these operators on the theta correspondences; first, their basic properties are given in $\$ 1$.

Similarly, a modular form on a subgroup of finite index in $\operatorname{SL}\left(2, \mathfrak{S}_{K}\right), K$ an imaginary quadratic field, is a vector valued function with domain the hyperbolic upper half space

$$
\mathfrak{Q}^{1}=\{x+y \mathbf{k} \text { quaternions } \mid x \in \mathbb{C}, 0<y \in \mathbb{R}\}
$$

(the homogeneous space of $\operatorname{SL}(2, \mathbb{C})$ ) satisfying properties analogous to (1)-(3) above. Since imaginary quadratic modular forms are less widely known, they are defined more fully and discussed in $\$ 2$ below.

Our first objective is to write down and study analogues of the Maass operators for these forms. This is accomplished in $\S 3$, which is independent of any theta series considerations. Namely, we introduce hyperbolic 3-space differential operators $\mathfrak{D}_{k}^{\mathrm{r}}$, $\mathfrak{D}_{k}^{1}$, which act on the modular forms for an imaginary quadratic field, raising and lowering (respectively) their weight $k$ by 2 . We give these operators both in terms of the universal enveloping algebra of the Lie algebra of $\operatorname{SL}(2, \mathbb{C})$, and by explicit formulas; these explicit formulas are more complicated than those for $\mathbf{D}_{k}^{\mathrm{r}}$ and $\mathbf{D}_{k}^{1}$, since the maximal compact $\mathrm{SU}(2, \mathbb{C})$ of $\operatorname{SL}(2, \mathbb{C})$ is not abelian. Though most of the properties of our operators are similar to those of the classical Maass operators, there are certain exceptions; for example, $\mathfrak{D}_{k}^{1}$ is either injective or identically zero $(k>2)$ [for fixed eigenvalues], while for $\mathbf{D}_{k}^{1}$ this is not true.

The remaining four sections relate theta series and the differential operators above. We focus first on the imaginary quadratic Doi-Naganuma (base change) lifting $\mathfrak{Z}$. The construction of this map, which associates to a modular form $f$ in $\{\Gamma(N), k, \lambda\}$ and a fixed imaginary quadratic field $K$ a modular form $\mathfrak{\&} f$ for $K$, is 
outlined in $\S 4$. Then, in $\S 5$, we show

THEOREM. The Maass operators and the hyperbolic upper half space operators are intertwined by the imaginary quadratic Doi-Naganuma (base change) lifting. More precisely, if $f$ is a modular form of weight $k$ and eigenvalue $\lambda$, then

$$
\begin{array}{ll}
\mathfrak{D}_{2 k}^{\mathrm{r}} \mathfrak{D}_{2 k-2}^{\mathrm{r}}(\mathfrak{L} f)=\mathfrak{L}\left(\mathbf{D}_{k}^{\mathrm{r}} f\right) & \text { for } k \geqslant 1, \\
\mathfrak{D}_{2 k-4}^{1} \mathfrak{D}_{2 k-2}^{1}(\mathfrak{L} f)=c(k, \lambda) \mathfrak{L}\left(\mathbf{D}_{k}^{1} f\right) & \text { for } k \geqslant 3,
\end{array}
$$

where $c(k, \lambda)$ is a constant (given below).

In a certain sense, (b) gives the imaginary quadratic analogue to the statement that holomorphic modular forms lift to holomorphic Hilbert modular forms under the real quadratic Doi-Naganuma lifting.

We also define a map $\mathfrak{L}_{-1}$ pulling modular forms for an imaginary quadratic field $K$ down to modular forms for $\mathbb{Q}$, and show that it too commutes with the action of these operators. Schematically, we have the commutativity of the diagram

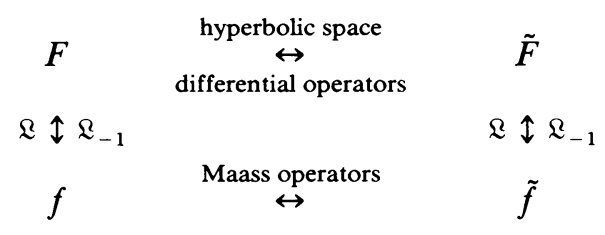

We give two proofs of this result. One looks at $L$-series and seems suitable to other base change contexts; the second studies the theta functions used as integral kernels to produce the lifting (cf. $\S 4$ and $[3,4]$ ). In this second proof, the diagrams are then consequences of a fundamental identity, which states roughly that the action of $\mathbf{D}_{k+2}^{1}$ on the $\mathfrak{G} \times \mathscr{G}^{1}$ theta kernel is the same as that of $\mathfrak{D}_{2 k}^{\mathrm{r}} \mathfrak{D}_{2 k-2}^{\mathrm{r}}$. Also, as an application, we show how to give the Doi-Naganuma lift of a (Maass) form of weight zero using the theta kernel. This case was previously problematic, since the kernel must be of weight at least one.

$\$ 6$ concerns the Shimura correspondence between modular forms of half integral and integral weight. We establish a result similar to the theorem above: applying one Maass operator on the half integral side corresponds to applying two to the related integral weight modular form. A related result may be found in Shimura [12, §2,6]. As an immediate corollary, we deduce the holomorphicity of the Shimura lift in weight greater than 3/2 (cf. Shimura [11], Cipra [2]). In $\$ 7$ we give similar results for the real quadratic Doi-Naganuma lifting, and a similar corollary.

Using the isogenies

$$
\mathrm{SO}(3, \mathbb{C}) \simeq \mathrm{SL}(2, \mathbb{C}), \quad \mathrm{SO}(4, \mathbb{C}) \simeq \operatorname{SL}(2, \mathbb{C}) \times \operatorname{SL}(2, \mathbb{C}),
$$

one may give Shimura type maps and quadratic liftings of imaginary quadratic modular forms as well (cf. [5]). It is reasonable to ask if results similar to those of $\S \S 6$ and 7, but using instead the hyperbolic 3-space differential operators, hold in these cases. They indeed do; however, we shall postpone the discussion of this to a future paper.

We remark that theta series correspondences have been studied from the point of view of representation theory by Howe, Rallis, Waldspurger, and others. Also, the 
existence of raising and lowering operators has been examined in the Hermitian case by M. Harris [6]. However, his methods do not apply to $\mathfrak{S}^{1}$, since it is non-Hermitian, nor do they give explicit differential operators formulas. We have chosen to work in a somewhat classical language-using modular forms instead of automorphic representations, and Whittaker functions instead of Whittaker models-so that we may be fully precise; accordingly, we are able to give exact formulas for the differential operators, and for their action on the Fourier coefficients of modular forms. We hope that this approach will be of interest.

1. Modular forms and Maass operators on the upper half plane. In this section we recall some properties of (nonholomorphic) modular forms and Maass operators. For a function $f(z)$ on $\mathfrak{B}$ and a matrix $\gamma=\left(\begin{array}{ll}a & b \\ c & d\end{array}\right)$ in $\mathrm{GL}^{+}(2, \mathbb{R})$, we define the weight $k$ slash operator

$$
\left(f \mid[\gamma]_{k}\right)(z)=(\operatorname{det} \gamma)^{k / 2}(c z+d)^{-k} f(\gamma z),
$$

where, as usual, $\gamma z=(a z+b)(c z+d)^{-1}$. The differential operator $-\Omega^{k,\left(2 k-k^{2}\right) / 4}$, defined in the introduction, corresponds to the Casimir operator

$$
\frac{1}{2}\left(\begin{array}{ll}
0 & 1 \\
0 & 0
\end{array}\right)\left(\begin{array}{ll}
0 & 0 \\
1 & 0
\end{array}\right)+\frac{1}{2}\left(\begin{array}{ll}
0 & 0 \\
1 & 0
\end{array}\right)\left(\begin{array}{ll}
0 & 1 \\
0 & 0
\end{array}\right)+\frac{1}{4}\left(\begin{array}{cc}
1 & 0 \\
0 & -1
\end{array}\right)^{2}
$$

of the universal enveloping algebra of $\operatorname{sl}(2, \mathbb{R})$, and hence $\Omega$ is invariant: for all $\gamma$ in $\mathrm{GL}^{+}(2, \mathbb{R})$ and twice continuously differentiable $f: \mathfrak{G} \rightarrow \mathbb{C}$,

$$
\Omega^{k, \lambda}\left(f \mid[\gamma]_{k}\right)=\left(\Omega^{k, \lambda} f\right) \mid[\gamma]_{k} .
$$

The shape of the Fourier expansion of a modular form $f$ in $\{\Gamma, k, \lambda\}$ is determined by the differential equation $\Omega^{k, \lambda} f=0$. Namely, for $\varepsilon= \pm 1, y>0, k, \lambda$ as above, set

$$
\begin{aligned}
\omega & =\left[(k-1)^{2}+4 \lambda\right]^{1 / 2} / 2, \\
u(y, k, \lambda) & = \begin{cases}y^{[(1-k) / 2-\omega]}, & \omega \neq 0, \\
y^{(1-k) / 2} \log y, & \omega=0,\end{cases}
\end{aligned}
$$

and

$$
W(\varepsilon y, k, \lambda)=y^{-k / 2} W_{\varepsilon k / 2, \omega}(2 y),
$$

where $W_{\varepsilon k / 2, \omega}$ is a Whittaker function (cf. [4]). Also, put

$$
\mathbf{e}[w]=\exp (2 \pi i w) \text {. }
$$

Then one has

Proposition 1.1. Let $f$ be in $\{\Gamma(N), k, \lambda\}$. Then $f$ has a Fourier expansion at the cusp corresponding to $A$ in $\operatorname{SL}(2, \mathbb{Z})$ given by

$$
\begin{aligned}
f \mid\left[A^{-1}\right]_{k}(z)= & c_{A}(0) y^{[(1-k) / 2+\omega]}+c_{A}^{\prime}(0) u(y, k, \lambda) \\
& +\sum_{n \neq 0} c_{A}(n) W\left(\frac{2 \pi n y}{N}, k, \lambda\right) \mathbf{e}\left[\frac{n x}{N}\right] .
\end{aligned}
$$


For such an $f$, and a (fixed) $A$ in $\operatorname{SL}(2, \mathbb{Z})$, let

$$
L_{+}(s, f)=\sum_{n=1}^{\infty} c_{A}(n) n^{-s}, \quad L_{-}(s, f)=\sum_{n=1}^{\infty} c_{A}(-n) n^{-s} .
$$

The Maass operators are defined by the formulas

$$
\mathbf{D}_{k}^{\mathrm{r}}=-\frac{1}{\pi}\left(2 i \frac{\partial}{\partial z}+k y^{-1}\right), \quad \mathbf{D}_{k}^{1}=-\frac{1}{\pi}\left(2 i y^{2} \frac{\partial}{\partial \bar{z}}\right)
$$

(so that in this case the lowering operator is independent of $k$ ). Their basic properties are given by

Proposition 1.2. (1) The Maass operators change weights by 2. That is, for any continuously differentiable $f: \mathfrak{Q} \rightarrow \mathbb{C}$ and $\gamma$ in $\mathrm{GL}^{+}(2, \mathbb{R})$,

$$
\begin{aligned}
\left(\mathbf{D}_{k}^{\mathrm{r}} f\right) \|[\gamma]_{k+2} & =\mathbf{D}_{k}^{\mathrm{r}}\left(f \mid[\gamma]_{k}\right) \quad \text { and }\left(\mathbf{D}_{k}^{1} f\right) \mid[\gamma]_{k-2}=\mathbf{D}_{k}^{1}\left(f \mid[\gamma]_{k}\right) . \\
\mathbf{D}_{k}^{\mathrm{r}} \Omega^{k, \lambda} & =\Omega^{k+2, \lambda-k} \mathbf{D}_{k}^{\mathrm{r}} \text { and } \mathbf{D}_{k}^{1} \Omega^{k, \lambda}=\Omega^{k-2, \lambda+k-2} \mathbf{D}_{k}^{1} .
\end{aligned}
$$

Thus the Maass operators take eigenfunctions of the Casimir operator to eigenfunctions.

$$
\Omega^{k, \lambda}=\pi^{2} \mathbf{D}_{k+2}^{1} \mathbf{D}_{k}^{\mathrm{r}}+\lambda-k=\pi^{2} \mathbf{D}_{k-2}^{\mathrm{r}} \mathbf{D}_{k}^{1}+\lambda .
$$

(4) $\mathbf{D}_{k}^{r}$ gives a linear map of $\{\Gamma(N), k, \lambda\}$ into $\{\Gamma(N), k+2, \lambda-k\}$ which is bijective for $\lambda \neq k$, and takes cusp forms to cusp forms.

$\mathbf{D}_{k}^{1}$ gives a linear map of $\{\Gamma(N), k, \lambda\}$ into $\{\Gamma(N), k-2, \lambda+k-2\}$ which is bijective for $\lambda \neq 0$, and takes cusp forms to cusp forms.

(5) The Maass operators shift Dirichlet series. Let $f$ be in $\{\Gamma(N), k, \lambda\}$. Then

$$
\begin{aligned}
& L_{+}\left(s, \mathbf{D}_{k}^{\mathrm{r}} f\right)=[2 / N] L_{+}(s-1, f) . \\
& L_{-}\left(s, \mathbf{D}_{k}^{\mathrm{r}} f\right)=[2(k-\lambda) / N] L_{-}(s-1, f) . \\
& L_{+}\left(s, \mathbf{D}_{k}^{1} f\right)=\left[-N \lambda /\left(2 \pi^{2}\right)\right] L_{+}(s+1, f) . \\
& L_{-}\left(s, \mathbf{D}_{k}^{1} f\right)=\left[N /\left(2 \pi^{2}\right)\right] L_{-}(s+1, f) .
\end{aligned}
$$

(6) $\mathbf{D}_{k}^{r}$ and $\mathbf{D}_{k}^{1}$ take Hecke eigenforms to Hecke eigenforms.

The proofs of the propositions above may be found in Maass [8] and Roelcke [10] (although with slightly different normalizations of the slash operator).

In addition, let

$$
\langle f, g\rangle=\int_{\Gamma(N) \backslash \mathscr{Q}} f(z) \overline{g(z)} y^{k-2} d x d y
$$

denote the Petersson inner product of two functions $f$ and $g$ of weight $k$ on $\Gamma(N)$ (with the usual assumptions on their growth to insure convergence).

Proposition 1.3. Suppose $f$ and $g$ are continuously differentiable functions on $\mathfrak{E}$ satisfying $f \mid[\gamma]_{k+2}=f$ and $g \mid[\gamma]_{k}=g$ for all $\gamma$ in $\Gamma(N)$. Suppose further that $f g$, $f \mathbf{D}_{k}^{\mathrm{r}} g$, and $g \mathbf{D}_{k+2}^{1} f$ are rapidly decreasing (in the sense of (2.16) of [12]). Then

$$
\left\langle\mathbf{D}_{k+2}^{1} f, g\right\rangle=\left\langle f, \mathbf{D}_{k}^{\mathrm{r}} g\right\rangle \text {. }
$$


This proposition is a special case of a result in Shimura [12]; it follows alternatively from an application of Green's theorem.

Finally, we recall the various Lie group formulations of $\mathbf{D}_{k}^{r}$ and $\mathbf{D}_{k}^{1}$. There is a one-to-one correspondence between functions $f: \mathbf{H} \rightarrow \mathbb{C}$ satisfying $(0.1)(1)$ and functions $f^{\sharp}: \operatorname{SL}(2, \mathbb{R}) \rightarrow \mathbb{C}$ such that

$$
f^{\#}(\gamma g \kappa(\theta))=e^{i k \theta} f^{\#}(g) \text { for all } \gamma \in \Gamma
$$

and

$$
\kappa(\theta)=\left(\begin{array}{cc}
\cos \theta & \sin \theta \\
-\sin \theta & \cos \theta
\end{array}\right) \in \mathrm{SO}(2)
$$

given by

$$
\begin{aligned}
& f^{\sharp}(g)=(c i+d)^{-k} f(g(i)), \\
& f(z)=(c i+d)^{k} f^{\sharp}(g) \quad \text { where } g=\left(\begin{array}{ll}
a & b \\
c & d
\end{array}\right) \text { and } g(i)=z .
\end{aligned}
$$

The elements

$$
X=\frac{1}{2}\left(\begin{array}{cc}
1 & i \\
i & -1
\end{array}\right) \text { and } Y=\frac{1}{2}\left(\begin{array}{cc}
1 & -i \\
-i & -1
\end{array}\right)
$$

of $\operatorname{sl}(2, \mathbb{C})$, the complexification of the (real) Lie algebra $\operatorname{sl}(2, \mathbb{R})$, satisfy

$$
\text { Ad } \kappa(\theta) X=e^{2 i \theta} X, \quad \text { Ad } \kappa(\theta) Y=e^{-2 i \theta} Y .
$$

A short computation then shows that, regarded as differential operators in the usual way (cf. also §2), the actions of $X$ and $Y$ on a function $f^{\sharp}$ give ( $-\pi$ times) the actions of $\mathbf{D}_{k}^{\mathbf{r}}$ and $\mathbf{D}_{k}^{1}$ on the corresponding $f$, respectively. Equivalently, let $V$ be an $(\operatorname{sl}(2, \mathbb{R}), \operatorname{SO}(2))$-module with weight vector $v$ of weight $k$ with respect to the action of $\mathbf{k}_{0}$ (= skew symmetric elements of $\operatorname{sl}(2, \mathbb{R})$ ). Complexify to get an $\mathrm{sl}(2, \mathbb{C})$-module. Then, when it is nonzero, $X v$ (respectively $Y v$ ) is a weight vector of weight $k+2$ (respectively $k-2$ ).

2. Modular forms on an imaginary quadratic field. Let $K$ be an imaginary quadratic field. The notion of a modular form on $K$ is based upon the action of $\operatorname{SL}(2, \mathbb{C})$ on $\mathfrak{S}^{1}$ by linear fractional transformation, taking representations of $\operatorname{SU}(2, \mathbb{C})$ into account. In this section we give these representations and the corresponding invariant Casimir operators explicitly, define modular forms on $K$, and describe their Fourier expansions. For proofs of the results here, see Friedberg [4].

For $n \geqslant 1$, let

$$
\left(\begin{array}{ll}
a & b
\end{array}\right)_{n}={ }^{\mathrm{t}}\left(\left(\begin{array}{l}
n \\
0
\end{array}\right) a^{n} \quad\left(\begin{array}{c}
n \\
1
\end{array}\right) a^{n-1} b \quad \cdots \quad\left(\begin{array}{l}
n \\
n
\end{array}\right) b^{n}\right),
$$

where ${ }^{\mathrm{t}}$ denotes the transpose. Define the $n$-fold symmetric power representation, $\sigma_{n}(g)$, for $g$ in $\operatorname{SL}(2, \mathbb{C})$, by

$$
\left(\left(\begin{array}{ll}
a & b
\end{array}\right)^{\mathrm{t}} g\right)_{n}=\sigma_{n}(g)\left(\begin{array}{ll}
a & b
\end{array}\right)_{n} .
$$

Equivalently, $\sigma_{n}(g)={ }^{\mathrm{t}} \tau_{n}\left({ }^{\mathrm{t}} g\right)$ where

$$
\left(\begin{array}{ll}
a & b
\end{array}\right)_{n}^{*}={ }^{\mathrm{t}}\left(\begin{array}{llll}
a^{n} & a^{n-1} b & \cdots & b^{n}
\end{array}\right) \text { and }\left(\left(\begin{array}{ll}
a & b
\end{array}\right)^{\mathrm{t} g}\right)_{n}^{*}=\tau_{n}(g)\left(\begin{array}{ll}
a & b
\end{array}\right)_{n}^{*} \text {. }
$$


The $\sigma_{n}(g)$ give irreducible $(n+1)$-dimensional representations of $\operatorname{SU}(2, \mathbb{C})$, and

$$
\operatorname{diag}\left(\left(\begin{array}{l}
n \\
0
\end{array}\right)^{1 / 2},\left(\begin{array}{l}
n \\
1
\end{array}\right)^{1 / 2}, \ldots,\left(\begin{array}{l}
n \\
n
\end{array}\right)^{1 / 2}\right)^{-1} \sigma_{n}(\kappa) \operatorname{diag}\left(\left(\begin{array}{l}
n \\
0
\end{array}\right)^{1 / 2},\left(\begin{array}{l}
n \\
1
\end{array}\right)^{1 / 2}, \ldots,\left(\begin{array}{l}
n \\
n
\end{array}\right)^{1 / 2}\right)
$$

is unitary for each $\kappa$ in $\operatorname{SU}(2, \mathbb{C})$. In addition, we set $\sigma_{0}(g)=1$ and $E_{n}=$ the $n \times \dot{n}$ identity.

Further, denote by $\rho$ the additive and multiplicative representation of the quaternions given by

$$
\rho(x+y \mathbf{k})=\left(\begin{array}{cc}
x & -y \\
\bar{y} & \bar{x}
\end{array}\right)
$$

for $x, y$ in $\mathbb{C}$, and for $g=\left(\begin{array}{ll}a & b \\ c & d\end{array}\right)$ in $\operatorname{SL}(2, \mathbb{C}), z$ in $\mathfrak{S}^{1}$, put $J(g, z)=\rho(c z+d)$. Consider $F: \mathfrak{S}^{1} \rightarrow \mathbb{C}^{k+1}(k \geqslant 0)$ a vector valued function on the hyperbolic upper half space, and $\gamma$ in $\operatorname{SL}(2, C)$. Then we define a slash operator of weight $k$ by

$$
\left(F \|[\gamma]_{k}\right)(z)=\sigma_{k}(J(\gamma, z))^{-1} F(\gamma z) .
$$

Let $\Gamma \subset \operatorname{SL}(2, \mathbb{C})$ be a group. Similarly to the $\operatorname{SL}(2, \mathbb{R})$ case, we have

Proposition 2.1. There is a one-to-one correspondence between

$$
\left\{F: \mathfrak{Q}^{1} \rightarrow \mathbb{C}^{k+1}|F| \|[\gamma]_{k}=F \text { for all } \gamma \in \Gamma\right\}
$$

and

$$
\left\{F^{\#:} \operatorname{SL}(2, \mathbb{C}) \rightarrow \mathbb{C}^{k+1} \mid F^{\sharp}(\gamma g \kappa)=\sigma_{k}\left({ }^{t} \kappa\right) F^{\#}(g) \text { for all } \gamma \in \Gamma, \kappa \in \operatorname{SU}(2, \mathbb{C})\right\}
$$

given by

$$
F(z)=\sigma_{k}(J(g, \mathbf{k})) F^{\sharp}(g) \quad \text { where } g \mathbf{k}=z,
$$

and

$$
F^{\sharp}(g)=\sigma_{k}(J(g, \mathbf{k}))^{-1} F(g \mathbf{k}) .
$$

To define a modular form it is necessary to introduce slash-invariant differential operators analogous to $\Omega$. Write $z$ in $\mathfrak{G}^{1}$ as $z=x+y \mathbf{k}(x$ in $\mathbb{C}, y>0$ in $\mathbb{R})$. We set

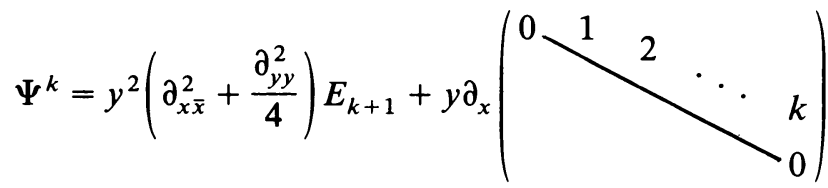

$$
\begin{aligned}
& +\frac{1}{4} y \partial_{y}\left(\begin{array}{cccc}
2 k-1 & & & \\
& 2 k-3 & & \\
& & \ddots & -1
\end{array}\right) \\
& +\frac{1}{4}\left(\begin{array}{llll}
k(k-2) & & & \\
& (k-1)(k-3) & & \\
& & \ddots & 0
\end{array}\right)
\end{aligned}
$$


and obtain $\tilde{\Psi}^{k}$ from $\Psi^{k}$ by replacing $(x, \bar{x})$ by $(-\bar{x},-x)$ and then conjugating by $\sigma_{k}\left(\begin{array}{ll}0 & 1 \\ 1 & 0\end{array}\right)$. Also, recall that an element $A$ of $\operatorname{sl}(2, \mathbb{C})$ gives rise to two differential operators $L_{A}, L_{A}^{\prime}$ :

$$
\left(L_{A} f\right)(g)=\left[\frac{\partial}{\partial t} f(g \exp (t A))\right]_{t=0}, \quad\left(L_{A}^{\prime} f\right)(g)=\left[\frac{\partial}{\partial t} f(g \exp (t A))\right]_{t=0} .
$$

We have

Proposition 2.2. (1) Let $F: \mathfrak{G}^{1} \rightarrow \mathbb{C}^{k+1}$ be twice continuously differentiable. Then

$$
\left(\Psi^{k} F\right)\left\|[g]_{k}=\Psi^{k}\left(F \|[g]_{k}\right), \quad\left(\tilde{\Psi}^{k} F\right)\right\|[g]_{k}=\tilde{\Psi}^{k}\left(F \|[g]_{k}\right)
$$

for all $\mathrm{g}$ in $\mathrm{SL}(2, \mathrm{C})$.

(2) $\Psi$ and $\tilde{\Psi}$ are the differential operators in the center of the universal enveloping algebra of $\mathrm{sl}(2, \mathbb{C})$ arising via $L, L^{\prime}$ (respectively) from

$$
\frac{1}{2}\left(\begin{array}{ll}
0 & 1 \\
0 & 0
\end{array}\right)\left(\begin{array}{ll}
0 & 0 \\
1 & 0
\end{array}\right)+\frac{1}{2}\left(\begin{array}{ll}
0 & 0 \\
1 & 0
\end{array}\right)\left(\begin{array}{ll}
0 & 1 \\
0 & 0
\end{array}\right)+\frac{1}{4}\left(\begin{array}{cc}
1 & 0 \\
0 & -1
\end{array}\right)^{2}
$$

( using the correspondence of Proposition 2.1), and they generate this center.

Thus it is reasonable to make (compare Weil [14])

Definition 2.3. A generalized Hilbert modular form for $K$ of weight $k$ and eigenvalues $\left(\lambda, \lambda^{\prime}\right)$ on a group $\Gamma \subset \operatorname{SL}\left(2, \mathfrak{D}_{K}\right)$ is a real analytic function $F: \mathfrak{S}^{1} \rightarrow \mathbb{C}^{k+1}$ satisfying:

(1) $F \|[\gamma]_{k}=F$ for all $\gamma$ in $\Gamma$;

(2) $F$ has (at worst) polynomial growth at each cusp;

(3) $\Psi^{k} F=\lambda F, \tilde{\Psi}^{k} F=\lambda^{\prime} F$.

We write the space of such forms $\left\{\left\{\Gamma, k,\left(\lambda, \lambda^{\prime}\right)\right\}\right\}$.

Note. In contrast to the $\operatorname{SL}(2, \mathbb{R})$ case described in $\S 1$, we take the eigenvalues here to be precisely those for the Casimir operators - there is no shift by $\left(2 k-k^{2}\right) / 4$.

Let $I$ be an ideal of $K, \mathrm{D}_{K}^{-1}$ be the inverse different, and

$$
\Gamma^{0}(I)=\left\{\left(\begin{array}{ll}
a & b \\
c & d
\end{array}\right) \in \operatorname{SL}\left(2, \mathfrak{D}_{K}\right) \mid b \in I\right\}
$$

(more generally, we could use any subgroup of $\operatorname{SL}\left(2, \mathfrak{D}_{K}\right)$ of finite index).

Proposition 2.4. Let $F$ be a modular form of weight $k$ and eigenvalues $\left(\lambda, \lambda^{\prime}\right)$ on a group $\Gamma^{0}(I)$. Then the Fourier expansion of $F(z)={ }^{\mathrm{t}}\left(F_{k}(z), \ldots, F_{0}(z)\right)$ is given by

$$
\begin{aligned}
F_{j}(z)= & \sum_{r \in\left(I \mathrm{D}_{K}\right)^{-1}-0}\left(\frac{r}{|r|}\right)^{j} C(r) Q_{j}\left(4 \pi y|r|, \lambda, \lambda^{\prime}\right) \mathbf{e}[\operatorname{tr}(r x)] \\
& +C_{1, j} y^{1-j+(4 \lambda+1)^{1 / 2}}+C_{2, j} q(y, j, \lambda)
\end{aligned}
$$

with

$$
q(y, j, \lambda)= \begin{cases}y^{1-j-(4 \lambda+1)^{1 / 2}}, & \lambda \neq-1 / 4 \\ y^{1-j} \log y, & \lambda=-1 / 4\end{cases}
$$

The Fourier expansion has a similar shape at the other cusps. 
Here the $Q_{j}\left(y, \lambda, \lambda^{\prime}\right)$ are sums of $K$-Bessel functions which can be computed by using the methods of [4]; for example,

$$
\begin{aligned}
& Q_{0}\left(y, \lambda, \lambda^{\prime}\right)=y K, \\
& Q_{1}\left(y, \lambda, \lambda^{\prime}\right)=-i\left(k y K^{\prime}+\left\{k^{2} / 2+2\left(\lambda-\lambda^{\prime}\right)\right\} K\right), \\
& Q_{2}\left(y, \lambda, \lambda^{\prime}\right)=-\left(\begin{array}{c}
k \\
2
\end{array}\right)\left\{y K^{\prime \prime}+\left(k+\frac{4\left(\lambda-\lambda^{\prime}\right)}{k}-1\right) K^{\prime}\right\} \\
& \quad+y^{-1} K\left\{\frac{k(1+4 \lambda)}{2}-\frac{k^{2}(k-2)^{2}}{8}-2\left(\lambda^{\prime}-\lambda\right)^{2}+\left(\lambda^{\prime}-\lambda\right)\left(k^{2}-2 k+2\right)\right\},
\end{aligned}
$$

where $K=K_{\sqrt{1+4 \lambda}}(y)$. (We shall find these explicit formulas for the first three components useful in the computations below.) We also set

$$
L(s, F, j)=\sum\left(\frac{r}{|r|}\right)^{j} C(r)(r \bar{r})^{-s}
$$

the twisted Dirichlet series for $F$, with $j$ in $\mathbb{Z}$.

Lastly, given $F: \mathfrak{S}^{1} \rightarrow \mathbb{C}^{k+1}$, we define an operator $\iota_{k}$ by

$$
\left(\iota_{k} F\right)(z)=\sigma_{k}\left(\begin{array}{ll}
0 & 1 \\
1 & 0
\end{array}\right) F(-\bar{z}) .
$$

PROPOSITION 2.5. $\iota_{k}$ is a bijective involution from the space of modular forms of weight $k$ and eigenvalues $\left(\lambda, \lambda^{\prime}\right)$ on $\Gamma^{0}(I)$ to the space of modular forms of weight $k$ and eigenvalues $\left(\lambda^{\prime}, \lambda\right)$ on $\Gamma^{0}(\bar{I})$, and takes cusp forms to cusp forms.

3. Differential operators on hyperbolic 3-space. With the notation continuing as above, we can now give the definition and basic properties of the hyperbolic raising and lowering operators.

DEFINITION 3.1. The hyperbolic raising operator of weight $k \geqslant 0, \mathfrak{D}_{k}^{\mathrm{r}}$, is the $(k+3) \times(k+1)$ matrix differential operator with entries

$$
\left(\mathfrak{D}_{k}^{\mathrm{r}}\right)_{i j}=\frac{1}{2 \pi \sqrt{-1}} \begin{cases}-\partial_{x}, & i=j, \\ \partial_{y}+k y^{-1}, & i=j+1, \\ \partial_{\bar{x}}, & i=j+2\end{cases}
$$

$(i=1, \ldots, k+3 ; j=1, \ldots, k+1)$ and all other entries zero.

The hyperbolic lowering operator of weight $k \geqslant 2, \mathfrak{D}_{k}^{1}$, is the $(k-1) \times(k+1)$ matrix differential operator with entries

$$
\left(\mathfrak{D}_{k}^{1}\right)_{i j}=\frac{1}{2 \pi \sqrt{-1}} \begin{cases}-\left(\begin{array}{c}
k-2 \\
i-1
\end{array}\right)\left(\begin{array}{c}
k \\
i-1
\end{array}\right)^{-1} y^{2} \partial_{\bar{x}}, & i=j, \\
\left(\begin{array}{c}
k-2 \\
i-1
\end{array}\right)\left(\begin{array}{c}
k \\
i
\end{array}\right)^{-1}\left(y^{2} \partial_{y}-y\right), & i=j-1, \\
\left(\begin{array}{c}
k-2 \\
i-1
\end{array}\right)\left(\begin{array}{c}
k \\
i+1
\end{array}\right)^{-1} y^{2} \partial_{x}, & i=j-2\end{cases}
$$

$(i=1, \ldots, k-1 ; j=1, \ldots, k+1)$ and all other entries zero. 
The binomial coefficients arise in the definition of $\mathfrak{D}_{k}^{1}$ because $\sigma_{k}$ is not unitary.

Let $\Gamma$ be a subgroup of $\operatorname{SL}\left(2, \mathfrak{D}_{K}\right.$ ) (of finite index). Put

$$
C\left(k, t, t^{\prime}\right)=\frac{-1}{(4 \pi)^{2}}\left(\begin{array}{l}
k \\
2
\end{array}\right)^{-1}\left(k^{2}\left(t+t^{\prime}\right)-2\left(t-t^{\prime}\right)^{2}+\frac{k^{2}}{2}-\frac{k^{4}}{8}\right) \text {. }
$$

Then we have

Proposition 3.2. (1) The hyperbolic differential operators change weights by 2. That is, for any continuously differentiable $F: \mathfrak{S}^{1} \rightarrow \mathbb{C}^{k+1}$ and $\gamma$ in $\operatorname{SL}(2, \mathbb{C})$,

$$
\begin{gathered}
\left(\mathfrak{D}_{k}^{\mathrm{r}} f\right)\left\|[\gamma]_{k+2}=\mathfrak{D}_{k}^{\mathrm{r}}\left(f \|[\gamma]_{k}\right), \quad\left(\mathfrak{D}_{k}^{1} f\right)\right\|[\gamma]_{k-2}=\mathfrak{D}_{k}^{1}\left(f \|[\gamma]_{k}\right) . \\
\mathfrak{D}_{k}^{\mathrm{r}} \Psi^{k}=\Psi^{k+2} \mathfrak{D}_{k}^{\mathrm{r}} \quad \text { and } \quad \mathfrak{D}_{k}^{\mathrm{r}} \tilde{\Psi}^{k}=\tilde{\Psi}^{k+2} \mathfrak{D}_{k}^{\mathrm{r}} . \\
\mathfrak{D}_{k}^{1} \Psi^{k}=\Psi^{k-2} \mathfrak{D}_{k}^{1} \quad \text { and } \quad \mathfrak{D}_{k}^{1} \tilde{\Psi}^{k}=\tilde{\Psi}^{k-2} \mathfrak{D}_{k}^{1} .
\end{gathered}
$$

Thus the hyperbolic differential operators take eigenfunctions of the Casimir operators to eigenfunctions.

$$
\mathfrak{D}_{k-2}^{\mathrm{r}} \mathfrak{D}_{k}^{1}=C\left(k, \Psi^{k}, \tilde{\Psi}^{k}\right), \quad \mathfrak{D}_{k+2}^{1} \mathfrak{D}_{k}^{\mathrm{r}}=C\left(k+2, \Psi^{k}, \tilde{\Psi}^{k}\right) .
$$

In particular, when $F$ is in $\left\{\left\{\Gamma, k,\left(\lambda, \lambda^{\prime}\right)\right\}\right\}, \mathfrak{D}_{k-2}^{\mathrm{r}} \mathfrak{D}_{k}^{1} F$ and $\mathfrak{D}_{k+2}^{1} \mathfrak{D}_{k}^{\mathrm{r}} F$ are multiples of $F$.

(4) $\mathfrak{D}_{k}^{\mathrm{r}}$ gives a linear map of $\left\{\left\{\Gamma, k,\left(\lambda, \lambda^{\prime}\right)\right\}\right\}$ into $\left\{\left\{\Gamma, k+2,\left(\lambda, \lambda^{\prime}\right)\right\}\right\}$ which is injective for $k>0$, has as kernel only the constant functions when $k=0$, is surjective when $C\left(k+2, \lambda, \lambda^{\prime}\right) \neq 0$, and takes cusp forms to cusp forms.

$\mathfrak{D}_{k}^{1}$ gives a linear map of $\left\{\left\{\Gamma, k,\left(\lambda, \lambda^{\prime}\right)\right\}\right\}$ into $\left\{\left\{\Gamma, k-2,\left(\lambda, \lambda^{\prime}\right)\right\}\right\}$ which is injective and surjective when $C\left(k, \lambda, \lambda^{\prime}\right) \neq 0$, zero when $C\left(k, \lambda, \lambda^{\prime}\right)=0$ (except when $k=2, \lambda=\lambda^{\prime}=0$, when its image is contained in $\mathbb{C}$ ), and takes cusp forms to cusp forms.

In particular,

$$
\operatorname{dim}_{\mathbb{C}}\left\{\left\{\Gamma, k,\left(\lambda, \lambda^{\prime}\right)\right\}\right\}=\operatorname{dim}_{\mathbb{C}}\left\{\left\{\Gamma, k+2,\left(\lambda, \lambda^{\prime}\right)\right\}\right\}
$$

when $C\left(k+2, \lambda, \lambda^{\prime}\right) \neq 0$.

(5) Let $F$ be in $\left\{\left\{\Gamma, k,\left(\lambda, \lambda^{\prime}\right)\right\}\right\}$. Then, with the notation of Proposition 2.4, if $F$ has Fourier coefficients $C(r)$, then $\mathfrak{D}_{k}^{\mathrm{r}} F$ has Fourier coefficients $C(r) \bar{r}$, and $\mathfrak{D}_{k}^{1} F$ has Fourier coefficients $C\left(k, \lambda, \lambda^{\prime}\right) C(r) / \bar{r}$. Thus

$$
\begin{aligned}
& L\left(s, \mathfrak{D}_{k}^{\mathrm{r}} F, j\right)=L\left(s-\frac{1}{2}, F, j-1\right), \\
& L\left(s, \mathfrak{D}_{k}^{1} F, j\right)=C\left(k, \lambda, \lambda^{\prime}\right) L\left(s+\frac{1}{2}, F, j+1\right),
\end{aligned}
$$

and the L-series at other cusps are similarly shifted.

(6) $\mathfrak{D}_{k}^{r}$ and $\mathfrak{D}_{k}^{1}$ take Hecke eigenforms to Hecke eigenforms.

$$
\iota_{k+2} \mathfrak{D}_{k}^{\mathrm{r}}=\mathfrak{D}_{k}^{\mathrm{r}} \iota_{k}, \quad \iota_{k-2} \mathfrak{D}_{k}^{1}=\mathfrak{D}_{k}^{1} \iota_{k} .
$$

Proof. It is sufficient to check (1) on the generators

$$
\left(\begin{array}{ll}
1 & c \\
0 & 1
\end{array}\right), \quad\left(\begin{array}{cc}
a & 0 \\
0 & a^{-1}
\end{array}\right), \quad \text { and } \quad\left(\begin{array}{cc}
0 & -1 \\
1 & 0
\end{array}\right)
$$


of $\operatorname{SL}(2, \mathbb{C})$, since $\left(F \|\left[\gamma_{1}\right]_{k}\right)\left\|\left[\gamma_{2}\right]_{k}=F\right\|\left[\gamma_{1} \gamma_{2}\right]_{k}$. For the first two types of generators, the statement is easily verified. As for the $\left(\begin{array}{ll}0 & -1 \\ 1 & 0\end{array}\right)$ case, observe that for any $f$ : $\mathfrak{Q}^{1} \rightarrow \mathbb{C}$,

$$
\left(\begin{array}{r}
-\partial_{x}\left(f\left(-z^{-1}\right)\right) \\
\partial_{y}\left(f\left(-z^{-1}\right)\right) \\
\partial_{\bar{x}}\left(f\left(-z^{-1}\right)\right)
\end{array}\right)=\sigma_{2}\left(-z^{-1}\right)\left(\begin{array}{r}
-\left(\partial_{x} f\right)\left(-z^{-1}\right) \\
\left(\partial_{y} f\right)\left(-z^{-1}\right) \\
\left(\partial_{\bar{x}} f\right)\left(-z^{-1}\right)
\end{array}\right) .
$$

The desired result then follows from an explicit computation.

The equalities of parts (2) and (3) may be verified by straightforward calculations. As for (4), the injectivity of $\mathfrak{D}_{k}^{r}, k>0$, and its 'almost injectivity' when $k=0$, is visible from its definition. The rest of the assertions there are then easily deduced from (1), (2), and (3). Alternatively, we may prove that $\mathfrak{D}_{k}^{1}$ is either injective or zero (or into $\mathbf{C}$ in the one special case) as follows. Given $F$ in $\left\{\left\{\Gamma, k,\left(\lambda, \lambda^{\prime}\right)\right\}\right\}$, we calculate the bottom entry in the column vector $\mathfrak{D}_{k}^{1} F$, using the explicit determination (2.1) of the $Q_{j}$ of Proposition 2.4 for $j=0,1,2$. One sees that

$$
\begin{gathered}
y\left[y Q_{0}(y)-\left(\begin{array}{l}
k \\
2
\end{array}\right)^{-1} y Q_{2}(y)+\frac{2 i}{k}\left\{Q_{1}(y)-y Q_{1}^{\prime}(y)\right\}\right] /(4 \pi)^{2} \\
=C\left(k, \lambda, \lambda^{\prime}\right) Q_{0}(y) .
\end{gathered}
$$

Since $\mathfrak{D}_{k}^{1} F$ is zero if (and only if) any component is zero, this gives the result.

Part (5) is similar. To find the Fourier coefficients of $\mathfrak{D}_{k}^{1} F$ and $\mathfrak{D}_{k}^{\mathrm{r}} F$, one computes the Fourier expansions of their bottom entries; the result for $\mathfrak{D}_{k}^{r}$ then follows at once from its definition, and that for $\mathfrak{D}_{k}^{1}$ from (3.1). Next, if $L(s, F, j)$ has an Euler product, so do its shifts by $\pm 1 / 2$; this implies (6). Alternatively, (6) follows from (1). And lastly, (7) is a consequence of the precise shapes of $\mathfrak{D}_{k}^{r}$ and $\mathfrak{D}_{k}^{1}$ - replacing $x$ by $-\bar{x}$ and then conjugating by $\sigma_{k}\left(\begin{array}{ll}0 & 1 \\ 1 & 0\end{array}\right)$ leaves them unchanged.

REMARK. In comparing Propositions 1.2 and 3.2 , the reader will note slight differences in the behavior of the Casimir eigenvalues under the differential operators. This is accounted for by the shift in eigenvalue built into the definition of $\Omega$, but not into $\Psi$ and $\tilde{\Psi}$ (cf. Note, $\S 2$ ).

As a first application of the raising and lowering operators, we see from part (5) above that the $L$-series $L(s, F, j)$, multiplied by suitable gamma factors, has a meromorphic continuation and functional equation, for each $j \leqslant k$ in $\mathbb{Z}$ (and for $j>k$ as well, when the lowering operators are nonzero), whose behavior can be read off directly from the Fourier expansion of $F$. In particular, this gives a simple alternate way to get the properties of the Dirichlet series $\Phi_{\alpha}(s)$ of Asai $[1, \S 3.6]$ for all $\alpha$.

Next, to define the Petersson inner product on $\mathfrak{S}^{1}$, let $M_{k}$ be the $(k+1) \times(k+1)$ diagonal matrix

$$
M_{k}=\operatorname{diag}\left(\left(\begin{array}{l}
k \\
0
\end{array}\right)^{-1},\left(\begin{array}{l}
k \\
1
\end{array}\right)^{-1}, \ldots,\left(\begin{array}{l}
k \\
k
\end{array}\right)^{-1}\right)
$$


( $M_{k}$ is needed because $\sigma_{k}$ is not unitary). Then given two functions $F, G: \mathfrak{S}^{1} \rightarrow \mathbb{C}^{k+1}$ of weight $k$ with respect to the group $\Gamma$ (i.e., satisfying Definition 2.3(1), (2)), one a cusp form, we put

$$
\langle\langle F, G\rangle\rangle=\int_{\Gamma \backslash \mathfrak{Q}^{1}}{ }^{\mathrm{t}} F(z) M_{k} \overline{G(z)} y^{k-3} d x d \bar{x} d y .
$$

This is well defined, for, as one can easily verify, $y^{-3} d x d \bar{x} d y$ is an invariant volume element, and, for any $\gamma=\left(\begin{array}{ll}a b \\ c & d\end{array}\right)$ in $\Gamma$,

$$
{ }^{\mathrm{t}} F(\gamma z) M_{k} \overline{G(\gamma z)}=|c z+d|^{2 k} F(z) M_{k} \overline{G(z)} .
$$

Proposition 3.3. Let $F$ and $G$ be functions of weights $k+2$ and $k$, respectively, with respect to the group $\Gamma$. Suppose that at least one of $F, G$ vanishes exponentially at each cusp of $\Gamma \backslash \mathfrak{S}^{1}$. Then

$$
\left\langle\left\langle\mathfrak{D}_{k+2}^{1} F, G\right\rangle\right\rangle=\left\langle\left\langle F, \mathfrak{D}_{k}^{\mathrm{r}} G\right\rangle\right\rangle .
$$

Proof. This follows from the Divergence Theorem, for the integrals over pieces of the boundary of $\Gamma \backslash \mathfrak{S}^{1}$ which are $\Gamma$-equivalent cancel. The estimates needed because of cusps are similar to those in Shimura [12, §2].

Recall that given $X=\left(\begin{array}{ll}0 & 1 \\ 0 & 0\end{array}\right)$ in $\operatorname{sl}(2, \mathbb{C})$, there are differential operators $L_{X}, L_{X}^{\prime}$ on $C^{\infty}(\operatorname{SL}(2, \mathbb{C}))$, and similarly for $Y=\left(\begin{array}{ll}0 & 0 \\ 1 & 0\end{array}\right), H=\left(\begin{array}{cc}1 & 0 \\ 0 & -1\end{array}\right)$ (see $\left.\S 2\right)$. We define the matrix differential operators $\left(\mathfrak{D}_{k}^{r}\right)^{\#},\left(\mathfrak{D}_{k}^{1}\right)^{\#}$, of dimensions $(k+3) \times(k+1),(k-1) \times(k$ $+1)$, respectively, by the formulas

$$
\begin{aligned}
& \left(\left(\mathfrak{D}_{k}^{\mathrm{r}}\right)^{\sharp}\right)_{i j}=\frac{1}{2 \pi \sqrt{-1}}\left\{\begin{array}{c}
-L_{X}, \quad i=j, \\
L_{H}, \quad i=j+1, \\
L_{Y}, \quad i=j+2,
\end{array}\right. \\
& \left(\left(\mathfrak{D}_{k}^{1}\right)^{\#}\right)_{i j}=\frac{1}{2 \pi \sqrt{-1}}\left\{\begin{array}{c}
-\left(\begin{array}{c}
k-2 \\
i-1
\end{array}\right)\left(\begin{array}{c}
k \\
i-1
\end{array}\right)^{-1} L_{X}^{\prime}, \quad i=j, \\
\left(\begin{array}{c}
k-2 \\
i-1
\end{array}\right)\left(\begin{array}{c}
k \\
i
\end{array}\right)^{-1} L_{H}^{\prime}, \quad i=j-1, \\
\left(\begin{array}{c}
k-2 \\
i-1
\end{array}\right)\left(\begin{array}{c}
k \\
i+1
\end{array}\right)^{-1} L_{Y}^{\prime}, \quad i=j-2,
\end{array}\right.
\end{aligned}
$$

where $i$ and $j$ run through the same values as in Definition 3.1 and all other entries are zero.

Proposition 3.4. $\left(\mathfrak{D}_{k}^{r}\right)^{\#}$ and $\left(\mathfrak{D}_{k}^{1}\right)^{\#}$ are the differential operators on functions $F^{\# \text { : }}$ $\operatorname{SL}(2, \mathbb{C}) \rightarrow \mathbb{C}^{k+1}$ which correspond to $\mathfrak{D}_{k}^{\mathrm{r}}, \mathfrak{D}_{k}^{1}$ on $F: \mathfrak{S}^{1} \rightarrow \mathbb{C}^{k+1}$ via Proposition 2.1. That is,

$$
\left(\mathfrak{D}_{k}^{\mathrm{r}} F\right)^{\#}=\left(\mathfrak{D}_{k}^{\mathrm{r}}\right)^{\sharp}\left(F^{\sharp}\right), \quad\left(\mathfrak{D}_{k}^{1} F\right)^{\#}=\left(\mathfrak{D}_{k}^{1}\right)^{\sharp}\left(F^{\#}\right) .
$$

Proof. One sees this by a straightforward calculation, whose details we omit.

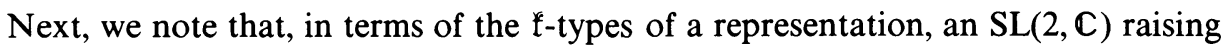
operator is natural. This was pointed out to me by Professor S. Kudla, and I thank 
him warmly for it. Let $(\pi, V)$ be a representation of $\operatorname{SL}(2, \mathbb{C})$. The induced representation $\pi_{*}$ of $g_{0}=\operatorname{sl}(2, \mathbb{C})$ extends to a representation of the complexified Lie algebra

$$
\mathfrak{g}=\mathfrak{g}_{0} \otimes_{\mathbf{R}} \mathbb{C}=\mathfrak{g}_{0} \oplus J \mathfrak{g}_{0} .
$$

Here $\mathfrak{g}_{0} \oplus J \mathfrak{g}_{0}$ is the complexification of a Cartan decomposition; $J$ reflects the integrable complex structure of the underlying manifold SL(2, C). Set $A=X+Y$, $B=X-Y, h=J H, S=A-i J B$, and take a highest weight vector $v$ in $V$ of weight $n$ with respect to

$$
\mathfrak{f}=(\mathbb{R} h+\mathbb{R} J A+\mathbb{R} B) \otimes_{\mathbb{R}} \mathbb{C} .
$$

Then an easy computation shows that $\pi_{*}(S) v$ is a highest weight vector of weight $n+2$ with respect to $f$. In fact, this construction goes through more generally-in any representation of the complexification of a real semisimple Lie algebra of compact type considered as a real Lie algebra, there will be raising operators on the $\mathfrak{f}$-types.

We close this section with the remark that we can define modular forms and raising and lowering operators for negative weight in hyperbolic space as well. Observe that in the $\operatorname{SL}(2, \mathbb{R})$ case, $f(z)$ is in $\{\Lambda, k, \lambda\}, \Lambda \subset \operatorname{SL}(2, \mathbb{Z})$, if and only if $y^{k} f(-\bar{z})$ is in $\{\Lambda,-k, \lambda-k\}$. In our case it is natural to consider an $F: \mathfrak{S}^{1} \rightarrow \mathbb{C}^{k+1}$, such that

$$
F(\gamma z)=\sigma_{k}\left({ }^{\mathrm{t}} J(\gamma, z)\right)^{-1} F(z) \text { for all } \gamma \text { in } \Gamma\left(\subseteq \operatorname{SL}\left(2, \mathfrak{D}_{K}\right)\right) \text {, }
$$

with appropriate growth conditions and differential equations, to be a modular form of weight $-k(<0)$ on $\Gamma$. With this notion, one sees that given $G$ of weight $k$ on $\Gamma, k$ any integer, then

$$
\left(\operatorname{inv}_{k} G\right)(z)=y^{k} \sigma_{|k|}\left(\begin{array}{cc}
1 & 0 \\
0 & -1
\end{array}\right) G(-\bar{z})
$$

is of weight $-k$. As for raising and lowering operators, we give them on forms $F$ of weight $-k \leqslant 0$ by

$$
\begin{aligned}
& \mathfrak{D}_{-k}^{\mathrm{r}}(F)=\operatorname{inv}_{k-2}\left(\mathfrak{D}_{k}^{1}\left(\operatorname{inv}_{-k} F\right)\right) \quad(-k<-1), \\
& \mathfrak{D}_{-k}^{1}(F)=\operatorname{inv}_{k+2}\left(\mathfrak{D}_{k}^{\mathrm{r}}\left(\operatorname{inv}_{-k} F\right)\right) ;
\end{aligned}
$$

this is analogous to the $\operatorname{SL}(2, \mathbb{R})$ case. The results above then carry over to negative weights as well with the obvious modifications.

4. The imaginary quadratic Doi-Naganuma lifting. Fix an imaginary quadratic field $K$. In this section we describe the Doi-Naganuma (base change) map $\mathfrak{L}$ for the extension $K / \mathbb{Q}$, taking modular forms for $\mathbb{Q}$ to forms for $K$, and also the map $\mathfrak{R}_{-1}$ pulling forms for $K$ down to those for $\mathbb{Q}$. We give $\mathfrak{L}$ and $\mathfrak{L}_{-1}$ explicitly as integration against an $\mathfrak{G} \times \mathfrak{G}^{1}$ theta series, which is constructed using an (indefinite) quadratic form of type $(3,1)$ naturally attached to $K$. This construction of the lifting $\mathfrak{Q}$, which is discussed in full detail in Friedberg [4], will be used in the next section in our proof of Theorem 5.1; an alternative construction, using the trace formula, may be found in the works of Saito, Shintani, and Langlands. 
Let $\mathfrak{B}$ denote the real 4-dimensional vector space

$$
\mathfrak{B}=\left\{\left.X \in M(2, \mathbb{C})\right|^{t} \bar{X}=X\right\},
$$

and let $Q$ be the quadratic form of type $(3,1)$ on $\mathfrak{B}$ defined by

$$
Q[X]=-2 \operatorname{det} X
$$

$\mathrm{SL}(2, \mathbb{C})$ acts on $\mathfrak{B}$ via $X^{g}={ }^{t} \bar{g} X g$. The majorants of $Q$ are the positive definite quadratic forms

$$
R^{g}[X]=\operatorname{tr}\left(\left(X^{g}\right)^{2}\right)
$$

for $g$ in $\operatorname{SL}(2, \mathbb{C}) . Q$ and $R^{g}$ will enter into the definition of the theta series. Next, we fix an ideal $I$ of $K$ and nonzero integers $M, P$, and consider the lattice

$$
\mathfrak{F}=\left\{\left(\begin{array}{cc}
m & r \\
\bar{r} & p
\end{array}\right) \in \mathfrak{B} \mid m \in M \mathbb{Z}, p \in P \mathbb{Z}, r \in I\right\} .
$$

Following Asai [1], we introduce the spherical harmonic polynomials of degree $k-1$,

$$
\eta_{k, \alpha}\left(\left(\begin{array}{cc}
m & r \\
\bar{r} & p
\end{array}\right)\right)=(k-1) ! \sum_{\beta, \gamma}(\alpha+\beta) !^{-1} \gamma !^{-1} 2^{-\beta} r^{\alpha} L_{\beta}^{(\alpha)}(2 r \bar{r}) H_{\gamma}(m-p),
$$

where $\alpha$ runs over integers with $|\alpha| \leqslant k-1, L$ and $H$ are Laguerre and Hermite polynomials, respectively (normalized as in [4, p. 487]), and the sum is over nonnegative integers $\beta$ and $\gamma$ such that $\alpha+2 \beta+\gamma=k-1$ and $\alpha+\beta \geqslant 0$. Finally, pick $V$ in $\mathfrak{F}^{*}$, the $Q$-dual of $\mathfrak{F}$.

Then the theta function of concern is defined by

$$
\begin{aligned}
& \boldsymbol{\theta}_{k, \alpha}(z, V, g, \mathfrak{F})=y^{1 / 2} \sum_{X \in \mathfrak{F}} \eta_{k, \alpha}\left((X-V)^{g}\right) \mathbf{e}\left[\frac{1}{2}\left(x Q+i y R^{g}\right)[X-V]\right], \\
& \boldsymbol{\theta}_{(k)}(z, V, g, \mathfrak{F})={ }^{\mathrm{t}}\left(\boldsymbol{\theta}_{k, 1-k}, \ldots, \boldsymbol{\theta}_{k, k-1}\right)
\end{aligned}
$$

with $z=x+i y$ in $\mathfrak{Q}$ and $g$ in $\operatorname{SL}(2, \mathbb{C})$. Set $-D=\operatorname{discrim}(K), N_{1}=N_{1}(\mathfrak{F})=$ 1.c.m. $(M P, D \cdot \operatorname{Norm}(I)), \chi_{-D}(n)=(-D / n)$, where $(-)$ is the Kronecker symbol, and

$$
G(\mathfrak{F})=\left\{g \in \operatorname{SL}(2, \mathbb{C}) \mid \mathfrak{F}^{g}=\mathfrak{F}\right\}
$$

The properties of this theta series are summarized in

Proposition 4.1. (1) For all $\sigma=\left(\begin{array}{ll}a & b \\ c & d\end{array}\right)$ in $\Gamma_{0}\left(N_{1}\right)$,

$$
\theta_{k, \alpha}(z, d V, g) \mid[\sigma]_{k}=\chi_{-D}(d) \theta_{k, \alpha}(z, V, g) \text {. }
$$

(2) For all $\kappa$ in $\mathrm{SU}(2, \mathbb{C})$ and $\gamma$ in $G(\mathfrak{F})$,

$$
\theta_{(k)}(z, V, \gamma g \kappa)=\sigma_{2 k-2}\left({ }^{\mathrm{t}} \kappa\right) \theta_{(k)}\left(z, V^{\gamma}, g\right) \text {. }
$$

$$
\Psi \theta_{k, \alpha}(z, V, g)=\tilde{\Psi} \theta_{k, \alpha}(z, V, g)=-\Omega^{k,\left(2 k-k^{2}\right) / 4} \theta_{k, \alpha}(z, V, g) \text {. }
$$

(We have dropped the $\mathfrak{F}$ to shorten the notation.) For the proofs, see Friedberg [3, 4]. 
We define $\mathfrak{L}$ and $\mathfrak{L}_{-1}$ by integrating against $\theta_{(k)}$. First, though, we sum over choices of the translation variable $V$. Let $\phi^{\prime}$ be a character of some subgroup $G^{\prime}(\mathfrak{F})$ of (finite index in) $G(\mathfrak{F}), S$ a finite subset of $\mathfrak{F}^{*}$ such that $S$ mod $\mathfrak{F}$ is fixed (as a set) by $G^{\prime}(\mathfrak{F})$, and $\phi: S \bmod \mathfrak{F} \rightarrow \mathbb{C}$ be such that

$$
\phi\left(V^{\gamma}\right)=\phi^{\prime}\left(\gamma^{-1}\right) \phi(V)
$$

for all $V$ in $S$ and $\gamma$ in $G^{\prime}(\mathfrak{F})$.

EXAMPLE. Let

$$
\phi^{\prime}\left(\left(\begin{array}{ll}
a & b \\
c & d
\end{array}\right)\right)=\chi \chi_{-D}(d \bar{d})
$$

where $\chi$ is a Dirichlet character mod MP (not necessarily primitive), and

$$
G^{\prime}(\mathfrak{F})=\left\{\left(\begin{array}{ll}
a & b \\
c & d
\end{array}\right) \in G(\mathfrak{F}) \mid b \in P I\right\}=G(\mathfrak{F}) \cap \Gamma^{0}(P I)
$$

Then if we set

$$
S=\left\{\left(\begin{array}{cc}
v / P & 0 \\
0 & 0
\end{array}\right) \mid v \in(\mathbb{Z} / M P \mathbb{Z})^{\times}\right\} \quad \text { and } \phi\left(\left(\begin{array}{cc}
v / P & 0 \\
0 & 0
\end{array}\right)\right)=\chi \chi_{-D}(v),
$$

the above conditions hold.

Put

$$
\boldsymbol{\theta}_{(k)}(z, g, \mathfrak{F}, S, \phi)=\sum_{V \in S} \phi(V) \theta_{(k)}(z, g, V, \mathfrak{F}) .
$$

(Mercifully, we shall often shorten the notation to $\theta_{(k)}(z, g)$ below.) Note that

$$
\theta_{(k)}\left(z, g^{\prime} g\right)=\phi^{\prime}\left(g^{\prime}\right) \theta_{(k)}(z, g) \text { for all } g^{\prime} \text { in } G^{\prime}(\mathfrak{F}) \text {. }
$$

Finally, let $f$ be a modular form of weight $k \geqslant 1$ and eigenvalue $\lambda$ on a group $\Gamma(N)$, a cusp form if $k=1$. Pick $\mathfrak{F}, S, \phi$ as above, so that $\theta_{(k)}(z, g, \mathfrak{F}, S, \phi)$ is $\Gamma(N)$ slash-invariant (for example, $N_{1}(\mathfrak{F}) \mid N$ is sufficient, but weaker conditions may do).

Definition 4.2. The Doi-Naganuma lift of $f$ is given by the Petersson inner product of $f$ with $\theta$ :

$$
(\mathfrak{L} f)_{\alpha}^{\#}(g)=\left\langle\theta_{k, \alpha}(z, g, \mathfrak{F}, S, \phi), f(z)\right\rangle, \quad(\mathfrak{L} f)^{\#}={ }^{\mathrm{t}}\left((\mathfrak{I} f)_{1-k}^{\#}, \ldots,(\mathfrak{L} f)_{k-1}^{\#}\right) .
$$

The Petersson inner product makes sense here by Proposition 4.1(1). As above, we let $\mathfrak{L} f$ be the function on $\mathfrak{S}^{1}$ corresponding to $(\mathfrak{L} f)^{\sharp}$ on $\operatorname{SL}(2, \mathbb{C})$ by Propositions 2.1 and 4.1(2).

THEOREM 4.3. If is a modular form for $K$ of weight $2 k-2$, and eigenvalue $\bar{\lambda}+\left(k^{2}-2 k\right) / 4$ with respect to both $\Psi$ and $\tilde{\Psi}$, on the group $G^{\prime}(\mathfrak{F}) \supset \Gamma_{\mathrm{SL}\left(2, \bigcirc_{K}\right)}(N)$. More precisely,

$$
(\mathfrak{f}) \|[\gamma]_{2 k-2}=\phi^{\prime}(\gamma)(\mathfrak{L}) \text { for all } \gamma \text { in } G^{\prime}(\mathfrak{F}) .
$$

Proof. This follows at once from Propositions 4.1(2) and (3), 1.2(3) and 1.3, and some computations.

REMARK. We shall extend the lift to the case of weight less than or equal to zero in $\S 5$ below. 
The actual computation of the integral which gives the lift is discussed in detail in Friedberg [3, 4]. For simplicity in stating the answer, we give here only the case corresponding to the example above. Then we have

THEOREM 4.4. Let $f$ be a cusp form of weight $k$ on $\Gamma_{0}(N)$ with character $\chi$ and eigenvalue $\lambda$. Then the Doi-Naganuma lift, corresponding to the lattice $\mathfrak{F}$ with $P=1$, $M=N$, is given by

$$
\begin{aligned}
(\Re f)_{\alpha}(x+y \mathbf{k})= & \sum_{t=1}^{\infty} t^{k-2}\left(\chi \chi_{-D}\right)(t) \sum_{r \in I^{-1} \mathrm{D}_{K}^{-1}-0}\left(\frac{\bar{r}}{|r|}\right)^{\alpha}|r|^{-1} \overline{b(N \cdot r \bar{r})} \\
& \cdot q_{|\alpha|}(t|r| y, \lambda) \mathbf{e}[\operatorname{tr}(r t x)]
\end{aligned}
$$

where

$$
f \mid\left[\begin{array}{cc}
0 & -1 \\
N & 0
\end{array}\right]_{k}=\sum b(n) W(2 \pi n y, k, \lambda) \mathbf{e}[n x]
$$

$\chi \chi_{-D}$ is a (not necessarily primitive) character $\bmod N$, and $q_{|\alpha|}$ is a certain sum of $K$-Bessel functions.

In particular, if $f$ is a Hecke eigenform, so is $\mathfrak{L} f$, and one Dirichlet series for the lifted form is (up to a constant)

$$
L(s, \mathfrak{R} f, k-1)=\sum \overline{b(n)} n^{-s} \sum \overline{b(n)} \chi_{-D}(n) n^{-s} .
$$

(Here, to get this Dirichlet series, we must sum over ideal classes if the class number of $K$ is greater than 1; see [4] for details.)

Lastly, we define in a similar way the map $\mathfrak{L}_{-1}$, given a modular form $F\left(z_{1}\right)$ for $K$ of weight $2 k-2$ and eigenvalues both $\bar{\lambda}+\left(k^{2}-2 k\right) / 4$ on a group $G^{\prime}(\mathfrak{F})$ as above, with $F$ a cusp form (cf. [4, Definition 2.4]) if $k=1$. Let $\theta_{(k)}\left(z, z_{1}\right)$ be the function on $\mathfrak{Q} \times \mathfrak{S}^{1}$ induced from $\theta_{(k)}(z, g)$ by Proposition 2.1 .

DEFINITION 4.5. The pull down of $F$ to a modular form for $\mathbb{Q}$ is given by

$$
\left(\mathfrak{R}_{-1} F\right)(z)=\left\langle\left\langle\theta_{(k)}\left(z, z_{1}\right), F\left(z_{1}\right)\right\rangle\right\rangle .
$$

This is analogous to the map studied by Shintani for the dual pair $(\operatorname{SL}(2, \mathbb{R})$, $\mathrm{SO}(2,1))$ in $[13]$.

Proposition 4.6. $\mathfrak{L}_{-1} F$ is a modular form of weight $k$ and eigenvalue $\lambda$ on $\Gamma(N)$.

The proof is similar to that of Theorem 4.3.

In the case of the example above, if

$$
F \|[\gamma]_{2 k-2}=\left(\chi \chi_{-D}\right)\left(d^{\prime} \overline{d^{\prime}}\right) F \quad \text { for all } \gamma=\left(\begin{array}{ll}
a^{\prime} & b^{\prime} \\
c^{\prime} & d^{\prime}
\end{array}\right) \text { in } G^{\prime}(\mathfrak{F})
$$

then

$$
\left(\mathfrak{L}_{-1} F\right) \mid[\sigma]_{k}=\chi(d)\left(\mathfrak{R}_{-1} F\right) \quad \text { for all } \sigma=\left(\begin{array}{ll}
a & b \\
c & d
\end{array}\right) \text { in } \Gamma_{0}\left(N_{1}\right)
$$


5. Differential operators and the imaginary quadratic Doi-Naganuma lifting. Fix a choice of lattice $\mathfrak{F}$, and $S, \phi$ as in $\S 4$ above, and modular forms $f$ for $\mathbb{Q}$ and $F$ for $K$ of the appropriate levels. Also, let

$$
c(k, \lambda)=\left(-16 \pi^{2}\right)^{-1}\left(\begin{array}{c}
2 k-2 \\
2
\end{array}\right)^{-1}\left(\begin{array}{c}
2 k-4 \\
2
\end{array}\right)^{-1}(k-1)^{2}(k-2)^{2}(4 \bar{\lambda}+2 k-3) .
$$

In this section we prove

THEOREM 5.1. (1) Let $f$ be a cusp form for $\mathbb{Q}$ of weight $k$ and eigenvalue $\lambda$. Then

$$
\begin{array}{ll}
\mathfrak{D}_{2 k}^{\mathrm{r}} \mathfrak{D}_{2 k-2}^{\mathrm{r}}(\mathfrak{L} f)=\mathfrak{L}\left(\mathbf{D}_{k}^{\mathrm{r}} f\right) & \text { for } k \geqslant 1, \\
\mathfrak{D}_{2 k-4}^{1} \mathfrak{D}_{2 k-2}^{1}(\mathfrak{L} f)=c(k, \lambda) \mathfrak{L}\left(\mathbf{D}_{k}^{1} f\right) & \text { for } k \geqslant 3 .
\end{array}
$$

(2) Let $F$ be a cusp form for $K$ of weight $2 k-2$ and eigenvalues $\bar{\lambda}+$ $\left(k^{2}-2 k\right) / 4$ for both $\Psi, \tilde{\Psi}$. Then

$$
\begin{array}{ll}
\mathfrak{L}_{-1}\left(\mathfrak{D}_{2 k}^{\mathrm{r}} \mathfrak{D}_{2 k-2}^{\mathrm{r}} F\right)=c(k+2, \bar{\lambda}-k) \mathbf{D}_{k}^{\mathrm{r}}\left(\mathfrak{L}_{-1} F\right) & \text { for } k \geqslant 1, \\
\mathfrak{L}_{-1}\left(\mathfrak{D}_{2 k-4}^{1} \mathfrak{D}_{2 k-2}^{1} F\right)=\mathbf{D}_{k}^{1}\left(\mathfrak{L}_{-1} F\right) & \text { for } k \geqslant 3 .
\end{array}
$$

In fact, these equalities hold for non cusp forms as well, as long as the integrals defining both sides converge.

Notice that, as an immediate consequence of (1)(b) and the injectivity of $\mathbb{R}$ on Hecke eigenforms, $c(k, \lambda)$ is never zero (for $\lambda$ in the discrete spectrum). By contrast, when $\lambda=0$, both sides of (1)(b) are identically zero (cf. Proposition 3.2 and Theorem 4.4).

FIRST PROOF OF PART 1 (FOR CUSP FORMS). This part follows from the computation of the lift $\mathfrak{Z}$ given in Theorem 4.4, and the calculation of the actions of the differential operators given in Propositions 1.2(5) and 3.2(5) (in the case of a more general lattice and form than that treated by Theorem 4.4, we must use Theorem 4.1 of [4] instead). The key observation here is that the operators $\mathbf{D}^{r}$ and $\mathbf{D}^{1}$ shift Dirichlet series by 1 , while $\mathfrak{D}^{\mathrm{r}}$ and $\mathfrak{D}^{1}$ shift by $1 / 2$ (in a suitable sense). Since the Dirichlet series before and after lift are related, this gives the theorem. We can thus expect a similar result in any GL(2) base change situation. We omit the details of this calculation.

SECOND PROOF (PARTS 1 AND 2). We shall obtain Theorem 5.1 by looking directly at the theta kernel. Philosophically speaking, properties of the Doi-Naganuma lifting ought to be fully explicable in terms of this theta kernel without needing to make the computation whose result is expressed by Theorem 4.4. The desired explanation is provided by

THEOREM 5.2.

$$
\begin{aligned}
& \text { (a) } \mathbf{D}_{k+2}^{1} \boldsymbol{\theta}_{(k+2)}(z, g)=\left(\mathfrak{D}_{2 k}^{\mathrm{r}}\right)^{\#}\left(\mathfrak{D}_{2 k-2}^{\mathrm{r}}\right)^{\#} \theta_{(k)}(z, g), \\
& \text { (b) }\left(\mathfrak{D}_{2 k+2}^{1}\right)^{\#} \boldsymbol{\theta}_{(k+2)}(z, g)=\frac{k+1}{4 k+2}\left(\mathfrak{D}_{2 k-2}^{\mathrm{r}}\right)^{\#} \mathbf{D}_{k}^{\mathrm{r}} \boldsymbol{\theta}_{(k)}(z, g)
\end{aligned}
$$

for all integers $k \geqslant 1$. 
Observe that part (a) implies parts (1)(a) and (2)(b) of Theorem 5.1 at once, by the adjointness properties given in Propositions 1.3 and 3.3. Parts (1)(b) and (2)(a) follow by applying $\left(\mathfrak{D}_{2 k}^{1}\right)^{\#}$ to both sides of (b), for the raising and lowering operators combine to give invariant differential operators which act as constants on $\mathfrak{L}\left(\mathbf{D}_{k}^{1} f\right)$ and $F$. We shall give a similar explanation for the commutativity of the differential operators with the Shimura and real quadratic Doi-Naganuma lifts below.

Proof of Theorem 5.2. We must do an explicit calculation, using (4.1). The action of the Maass operators on the theta kernel is easy to compute. As for $\left(\mathfrak{D}_{k}^{r}\right)^{\#}$ and $\left(\mathfrak{D}_{k}^{1}\right)^{\#}$, pick $X$ in $\mathfrak{B}$, and embed $C^{\infty}(\mathfrak{B})$ in $C^{\infty}(\operatorname{SL}(2, \mathbb{C}))$ by associating to $h$ in $C^{\infty}(\mathfrak{B})$ the function $h_{X}(g)=h\left(X^{g}\right)$. Then using Proposition 3.4, one sees that the operator $\left(\mathfrak{D}_{k}^{\mathrm{r}}\right)^{\#}$ induces an operator $\left(\mathfrak{D}_{k}^{\mathrm{r}}\right)_{0}^{\#}: C^{\infty}(\mathfrak{B})^{k+1} \rightarrow C^{\infty}(\mathfrak{B})^{k+3}$ such that

$$
\left(\left(\mathbf{D}_{k}^{\mathrm{r}}\right)_{0}^{\#} h\right)_{X}(g)=\left(\mathfrak{D}_{k}^{\mathrm{r}}\right)^{\sharp}\left(h_{X}(g)\right)
$$

for each $X$ in $\mathfrak{B}$ and $h$ in $C^{\infty}(\mathfrak{B})^{k+1}$; with the usual parametrization

$$
\mathfrak{B}=\left\{\left(\begin{array}{cc}
m & r \\
\bar{r} & p
\end{array}\right)\right\}
$$

$\left(\mathfrak{D}_{k}^{\mathrm{r}}\right)_{0}^{\#}$ is given by

$$
\left(\left(\mathfrak{D}_{k}^{\mathrm{r}}\right)_{0}^{\#}\right)_{i j}=\frac{1}{2 \pi \sqrt{-1}} \begin{cases}-\left(m \frac{\partial}{\partial r}+\bar{r} \frac{\partial}{\partial p}\right), & i=j, \\ m \frac{\partial}{\partial m}-r \frac{\partial}{\partial r}+\bar{r} \frac{\partial}{\partial \bar{r}}-p \frac{\partial}{\partial p}, & i=j+1, \\ r \frac{\partial}{\partial m}+p \frac{\partial}{\partial \bar{r}}, & i=j+2 .\end{cases}
$$

An operator $\left(\mathfrak{D}_{k}^{1}\right)_{0}^{\#}: C^{\infty}(\mathfrak{B})^{k+1} \rightarrow C^{\infty}(\mathfrak{B})^{k-1}$, given by

$$
\left(\left(\mathfrak{D}_{k}^{1}\right)_{0}^{\#}\right)_{i j}=\frac{1}{2 \pi \sqrt{-1}} \begin{cases}-\left(\begin{array}{c}
k-2 \\
i-1
\end{array}\right)\left(\begin{array}{c}
k \\
i-1
\end{array}\right)^{-1}\left(m \frac{\partial}{\partial \bar{r}}+r \frac{\partial}{\partial p}\right), & i=j, \\
\left(\begin{array}{c}
k-2 \\
i-1
\end{array}\right)\left(\begin{array}{c}
k \\
i
\end{array}\right)^{-1}\left(m \frac{\partial}{\partial m}+r \frac{\partial}{\partial r}-\bar{r} \frac{\partial}{\partial \bar{r}}-p \frac{\partial}{\partial p}\right), & i=j-1, \\
\left(\begin{array}{c}
k-2 \\
i-1
\end{array}\right)\left(\begin{array}{c}
k \\
i+1
\end{array}\right)^{-1}\left(\bar{r} \frac{\partial}{\partial m}+p \frac{\partial}{\partial r}\right), & i=j-2,\end{cases}
$$

is similarly defined. However, $\theta_{(k)}(z, g)$ is in the sum over $X$ in $\mathfrak{F}^{*}$ of the spaces $C^{\infty}(\mathfrak{B})_{X}^{2 k-1}$. Thus, we can calculate both sides of (5.1) (a) and (b) directly from (4.1) by using the formulas above. Comparing these calculations completes the proof [notice that it suffices to check equality for only one component in each equation, by the irreducibility of $\sigma_{k}$ ].

This proof may be summarized by saying that the spherical functions $\eta_{k, \alpha}$ are built out of the action of the differential operators; in essence, this is true because all objects in (5.1) are canonical.

REMARK. One can also derive part (b) of Theorem 5.2 from part (a) by applying $\mathbf{D}_{k}^{\mathrm{r}}\left(\mathfrak{D}_{2 k+2}^{1}\right)^{\#}$ to both sides, and then using Propositions 1.2, 3.2, and 4.1. 
We close this section by noting an application of the differential operators and Theorem 5.1.

ApPlication 5.3. We can use the theta kernel to lift modular forms $f$ of weight 0 , even though $\theta_{(k)}$ is defined only for weight $k \geqslant 1$. Namely, we must apply $\mathbf{D}_{0}^{\mathrm{r}}$, raising the weight to 2 , lift (by integrating against $\theta_{(2)}$ ), and then reduce the weight twice, using $\mathfrak{D}_{0}^{1} \mathfrak{D}_{2}^{1}$, to get a form of weight -2 . Note that the $L$-series before and after lift will thus correspond in the usual base change way. (In fact, weights 2 and -2 are equivalent, as indicated in §3.) By Theorem 5.1, if we apply $\mathbf{D}_{2}^{\mathrm{r}} \mathbf{D}_{0}^{\mathrm{r}}$ before lifting, to get a form of weight 4 , integrate against $\theta_{(4)}$, and then apply $\mathfrak{D}_{0}^{1} \mathfrak{D}_{2}^{1} \mathfrak{D}_{4}^{1} \mathfrak{D}_{6}^{1}$, we get (up to a constant) the same answer for the lifted form as by the procedure above, and similarly if we raise more than twice before lifting. This procedure also allows us to lift noncusp forms of weight one.

6. Differential operators and the Shimura correspondence. We consider next the Shimura correspondence (Shimura [11], Shintani [13]), which gives a map between modular forms of weight $\kappa / 2(\kappa \geqslant 1$ odd) and forms of weight $\kappa-1$. For convenience, we shall treat modular forms only over $\mathbb{Q}$; the extension to forms over an arbitrary totally real field is immediate. Our results are (a) theorems analogous to those of $\$ 5$ and, as an immediate corollary of these, (b) a simple proof that, for $\kappa>3$, holomorphic forms go to holomorphic forms under the Shimura maps. This corollary thus gives an easy alternative to the growth estimate and Poincaré series methods of Niwa [9] and Cipra [2], previously used to prove holomorphicity.

First, we briefly recall the theta kernel approach to the Shimura correspondence, based upon the reductive dual pair $(\operatorname{SL}(2, \mathbb{R}), \operatorname{SO}(2,1))$ (see Niwa [9], Shintani [13]). Let

$$
\mathfrak{B}_{0}=\left\{X=\left(\begin{array}{cc}
x_{1} & x_{2} / 2 \\
x_{2} / 2 & x_{3}
\end{array}\right) \mid x_{1}, x_{2}, x_{3} \in \mathbb{R}\right\}
$$

be a real 3-dimensional vector space, with $\operatorname{SL}(2, \mathbb{R})$ action given by $X^{g}={ }^{t} g X g$. Then, using the quadratic form of type $(2,1)$ on $\mathfrak{B}_{0}$,

$$
Q[X]=-2 \operatorname{det} X
$$

with majorants given for $g$ in $\operatorname{SL}(2, \mathbb{R})$ by

$$
R^{g}[X]=\operatorname{tr}\left(\left(X^{g}\right)^{2}\right)
$$

and a suitable spherical function, $\left(2 i\left(x_{1}+i x_{2}-x_{3}\right)\right)^{(\kappa-1) / 2}$, analogous to $\left\{\eta_{k, \alpha}\right\}$, one can form a theta kernel $\theta_{(\kappa / 2)}\left(z, z^{\prime}\right)$ on $\mathfrak{G} \times \mathfrak{G}$, of weight $\kappa / 2$ in the first variable, and $\kappa-1$ in the second variable. This construction is similar to (4.2), (4.3) above. Taking the Petersson inner product with $\theta$ in one of the variables gives the Shimura correspondences, with $L$-series before and after lift related (cf. Shimura [11], Shintani [13]). We denote these maps by $\subseteq(\kappa / 2$ to $\kappa-1)$ and $\subseteq_{-1}(\kappa-1$ to 
$\kappa / 2)$. Put $c_{1}(\kappa / 2, \lambda)=4 \pi^{-2}(3-\kappa-4 \bar{\lambda})$. Then, with $f$ and $F$ forms of the appropriate levels, we have

THEOREM 6.1. (1) Let $f$ be a cusp form of weight $\kappa / 2$ and eigenvalue $\lambda$. Then

$$
\begin{aligned}
& \mathbf{D}_{\kappa+1}^{r} \mathbf{D}_{\kappa-1}^{r}(\Im f)=\Im\left(\mathbf{D}_{\kappa / 2}^{\mathrm{r}} f\right) \text { for } \kappa \geqslant 1 . \\
& \mathbf{D}_{\kappa-3}^{1} \mathbf{D}_{\kappa-1}^{1}(\Im f)=c_{1}(\kappa / 2, \lambda) \Im\left(\mathbf{D}_{\kappa / 2}^{1} f\right) \text { for } \kappa>3 .
\end{aligned}
$$

(2) Let $F$ be a cusp form of weight $\kappa-1$ and eigenvalue $4 \bar{\lambda}$. Then

(a) $\quad \mathfrak{S}_{-1}\left(\mathbf{D}_{\kappa+1}^{\mathrm{r}} \mathbf{D}_{\kappa-1}^{\mathrm{r}} F\right)=c_{1}(\kappa / 2+2, \bar{\lambda}-\kappa / 2) \mathbf{D}_{\kappa / 2}^{\mathrm{r}}\left(\mathfrak{S}_{-1} F\right)$ for $\kappa \geqslant 1$.

(b) $\varsigma_{-1}\left(\mathbf{D}_{\kappa-3}^{1} \mathbf{D}_{\kappa-1}^{1} F\right)=\mathbf{D}_{\kappa / 2}^{1}\left(\Im_{-1} F\right)$ for $\kappa>3$.

As with Theorem 5.1, the result holds for non cusp forms too, provided both sides are defined.

As an immediate corollary, we get

COROLlaRY 6.2. The Shimura correspondences $\mathbb{S}, \mathfrak{S}_{-1}$ take holomorphic forms to holomorphic forms $(\kappa>3)$.

Proof. This follows from parts (1)(b) and (2)(b) of Theorem 6.1, since the lowering operator $\mathbf{D}^{1}$ annihilates holomorphic forms.

We remark that this result can be extended to the case $\kappa=3$ as well. It is false for $\kappa=1$ (Cipra [2]).

Lastly, we give two theta kernel identities from which Theorem 6.1 may be immediately deduced. For convenience, let us write differential operators on the second variable with primes.

THEOREM 6.3. Let $\kappa$ be an odd positive integer. Then

$$
\begin{gathered}
\mathbf{D}_{\kappa / 2+2}^{1} \boldsymbol{\theta}_{(\kappa / 2+2)}\left(z, z^{\prime}\right)=\left(\mathbf{D}_{\kappa+1}^{\mathrm{r}}\right)^{\prime}\left(\mathbf{D}_{\kappa-1}^{\mathrm{r}}\right)^{\prime} \boldsymbol{\theta}_{(\kappa / 2)}\left(z, z^{\prime}\right) . \\
16 \pi^{-2} \mathbf{D}_{\kappa / 2}^{\mathrm{r}} \boldsymbol{\Omega}^{\kappa / 2,(\kappa-1) / 4} \boldsymbol{\theta}_{(\kappa / 2)}\left(z, z^{\prime}\right)=\left(D_{\kappa+1}^{1}\right)^{\prime}\left(D_{\kappa+3}^{1}\right)^{\prime} \boldsymbol{\theta}_{(\kappa / 2+2)}\left(z, z^{\prime}\right) .
\end{gathered}
$$

The proof is similar to that of Theorem 5.2. We suppress the details, except to note that on $C^{\infty}\left(\mathfrak{B}_{0}\right)$ the raising operator $\left(\mathbf{D}^{\mathrm{r}}\right)^{\prime}$ (resp. the lowering operator $\left.\left(\mathbf{D}^{1}\right)^{\prime}\right)$ corresponds to the action of

$$
-\frac{1}{\pi}\left[x_{1} \frac{\partial}{\partial x_{1}}-x_{3} \frac{\partial}{\partial x_{3}}+i\left(x_{1}+x_{3}\right) \frac{\partial}{\partial x_{2}}+\frac{i x_{2}}{2}\left(\frac{\partial}{\partial x_{1}}+\frac{\partial}{\partial x_{3}}\right)\right]
$$

(resp. the complex conjugate of (6.1)), under the embedding of $C^{\infty}\left(\mathfrak{B}_{0}\right)$ in $C^{\infty}(\operatorname{SL}(2, \mathbb{R}))$ given by $h_{X}(g)=h\left(X^{g}\right)$.

7. Differential operators and the real quadratic Doi-Naganuma lifting. Using a quadratic form of type $(2,2)$, one can construct a theta series $\theta_{(k)}\left(z, z_{1}, z_{2}\right)$ on $\mathfrak{S} \times \mathfrak{G} \times \mathfrak{E}$, similar to those above, which gives the real quadratic Doi-Naganuma lifting $\mathfrak{L}^{\mathbf{k}}$. $\mathfrak{L}^{\mathbf{R}}$ assigns to a modular form $f$ of weight $k$ and eigenvalue $\lambda$ and a real quadratic field $K$ a Hilbert modular form $\mathfrak{L}^{\mathbf{R}} f$ of weight $(k, k)$ and eigenvalues $(\bar{\lambda}, \bar{\lambda})$ for $K$ (cf. Kudla [7] for the holomorphic case); there is also a map $\mathfrak{Q}_{-1}^{\mathbf{R}}$, similar to $\mathfrak{L}_{-1}$ of $\S 4$. In this context, theorems like those of $\$ \S 5$ and 6 hold. Namely, denote differential operators on the $z_{1}$ and $z_{2}$ variables with superscripts ${ }^{(1)}$ and ${ }^{(2)}$. 
Then we have

THEOREM 7.1. (1) Let $f$ be a cusp form of weight $k$ and eigenvalue $\lambda$. Then

$$
\begin{array}{ll}
\left(\mathbf{D}_{k}^{\mathbf{r}}\right)^{(1)}\left(\mathbf{D}_{k}^{\mathbf{r}}\right)^{(2)}\left(\mathfrak{Q}^{\mathbf{R}} f\right)=\mathfrak{Q}^{\mathbf{R}}\left(\mathbf{D}_{k}^{\mathrm{r}} f\right) & \text { for } k \geqslant 0 . \\
\left(\mathbf{D}_{k}^{1}\right)^{(1)}\left(\mathfrak{Q}^{\mathbf{R}} f\right)=\left(\mathbf{D}_{k-2}^{\mathrm{r}}\right)^{(2)} \mathfrak{Q}^{\mathbf{R}}\left(\mathbf{D}_{k}^{1} f\right) & \text { for } k \geqslant 2 . \\
\left(\mathbf{D}_{k}^{1}\right)^{(2)}\left(\mathfrak{Q}^{\mathbf{R}} f\right)=\left(\mathbf{D}_{k-2}^{\mathrm{r}}\right)^{(1)} \mathfrak{Q}^{\mathbf{R}}\left(\mathbf{D}_{k}^{1} f\right) & \text { for } k \geqslant 2 .
\end{array}
$$

(2) Let $F$ be a Hilbert modular form of weight $(k, k)$ and eigenvalues $(\bar{\lambda}, \bar{\lambda})$. Then

$$
\begin{array}{ll}
\mathfrak{Q}_{-1}^{\mathbf{R}}\left(\left(\mathbf{D}_{k}^{\mathbf{r}}\right)^{(1)}\left(\mathbf{D}_{k}^{\mathbf{r}}\right)^{(2)} F\right)=\frac{k-\lambda}{\pi^{2}} \mathbf{D}_{k}^{\mathrm{r}}\left(\mathfrak{Q}_{-1}^{\mathbf{R}} F\right) & \text { for } k \geqslant 0 . \\
\mathfrak{L}_{-1}^{\mathbf{R}}\left(\left(\mathbf{D}_{k}^{1}\right)^{(1)}\left(\mathbf{D}_{k}^{1}\right)^{(2)} F\right)=\mathbf{D}_{k}^{1}\left(\mathfrak{Q}_{-1}^{\mathbf{R}} F\right) & \text { for } k \geqslant 2 .
\end{array}
$$

COROLlaRY 7.2. The real quadratic Doi-Naganuma correspondences $\mathfrak{L}^{\mathbf{R}}$, $\mathfrak{L}_{-1}^{\mathbf{R}}$ take holomorphic forms to holomorphic forms $(k \geqslant 2)$.

THEOREM 7.3. Let $k$ be a nonnegative integer. Then

$$
\begin{aligned}
& \text { (1) } \mathbf{D}_{k+2}^{1} \boldsymbol{\theta}_{(k+2)}\left(z, z_{1}, z_{2}\right)=\left(\mathbf{D}_{k}^{\mathrm{r}}\right)^{(1)}\left(\mathbf{D}_{k}^{\mathrm{r}}\right)^{(2)} \boldsymbol{\theta}_{(k)}\left(z, z_{1}, z_{2}\right) . \\
& \text { (2) }\left(\mathbf{D}_{k+2}^{1}\right)^{(1)} \boldsymbol{\theta}_{(k+2)}\left(z, z_{1}, z_{2}\right)=\left(\mathbf{D}_{k}^{\mathrm{r}}\right)^{(2)} \mathbf{D}_{k}^{\mathrm{r}} \boldsymbol{\theta}_{(k)}\left(z, z_{1}, z_{2}\right) . \\
& \text { (3) }\left(\mathbf{D}_{k+2}^{1}\right)^{(2)} \boldsymbol{\theta}_{(k+2)}\left(z, z_{1}, z_{2}\right)=\left(\mathbf{D}_{k}^{\mathrm{r}}\right)^{(1)} \mathbf{D}_{k}^{\mathrm{r}} \boldsymbol{\theta}_{(k)}\left(z, z_{1}, z_{2}\right) .
\end{aligned}
$$

The proofs of these results are similar to those given above.

\section{BIBLIOGRAPHY}

1. T. Asai, On the Doi-Naganuma lifting associated with imaginary quadratic fields, Nagoya Math. J. 71 (1978), 149-167.

2. B. Cipra, On the Niwa-Shintani theta-kernel lifting of modular forms, Nagoya Math. J. 91 (1983), 49-117.

3. S. Friedberg, On the imaginary quadratic Doi-Naganuma lifting of modular forms of arbitrary level, Nagoya Math. J. 92 (1983), 1-20.

4. On Maass wave forms and the imaginary quadratic Doi-Naganuma lifting, Math. Ann. 263 (1983), 483-508.

5. Theta series correspondences and modular forms for number fields, Modular Forms and Functions (R. A. Rankin, ed.) (to appear).

6. M. Harris, Maass operators and Eisenstein series, Math. Ann. 258 (1981), 135-144.

7. S. Kudla, Theta functions and Hilbert modular forms, Nagoya Math. J. 69 (1978), 97-106.

8. H. Maass, Lectures on modular functions of one complex variable, Tata Inst. Fund. Res., 1964.

9. S. Niwa, Modular forms of half integral weight and the integral of certain theta-functions, Nagoya Math. J. 56 (1974), 147-161.

10. W. Roelcke, Das Eigenwertproblem der automorphen Formen in der hyperbolischen Ebene. I, II, Math. Ann. 167 (1966), 292-337; 168 (1967), 261-324.

11. G. Shimura, On modular forms of half integral weight, Ann. of Math. (2) 97 (1973), 440-481.

12. _ On certain zeta functions attached to two Hilbert modular forms. I: The case of Hecke characters, Ann. of Math. (2) 114 (1981), 127-164.

13. T. Shintani, On construction of holomorphic cusp forms of half integral weight, Nagoya Math. J. 58 (1975), 83-126.

14. A. Weil, Dirichlet series and automorphic forms, Lecture Notes in Math., vol. 189, Springer, 1971.

Department of Mathematics, Harvard University, Cambridge, Massachusetts 02138 